\title{
Frozen shoulder or missed posterior dislocation?
}

\author{
D Leijnen, ${ }^{1,2}$ MD, MMed (Sports Med); J T Viljoen, ${ }^{1}$ BSc (Physio), MPhil (Exercise Sci); J H Kirby, ${ }^{1}$ MB ChB, MSc (Sports Med); \\ P L Viviers, ${ }^{1} \mathrm{MB}$ ChB, MMed Sc, MSc (Sports Med)
}

${ }^{1}$ Campus Health Service and the Centre for Human Performances Sciences, Stellenbosch University, Stellenbosch, South Africa

${ }^{2}$ Department of Physical Medicine and Rehabilitation, Catholic University of Leuven, Leuven, Belgium

Corresponding author: P L Viviers (plv@sun.ac.za)

The clinical presentation of a missed posterior shoulder dislocation has similarities with an idiopathic frozen shoulder masking proper diagnosis at the time of injury. We report a case of a 48-year-old woman with shoulder pain which demonstrates the importance of correct initial diagnosis and management.

S Afr J Sports Med 2015;27(2):58-59. DOI:10.7196/SAJSM.613

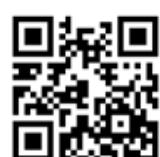

The glenohumeral (GH) joint consists of the humeral head that articulates in the glenoid fossa of the scapula, surrounded by several static and dynamic structures. It is the most mobile joint of the human body which makes it vulnerable to dislocation in all directions. Posterior shoulder dislocation (PSD) is less common than anterior. The rarity of this pathology together with the restrictions of standard view $\mathrm{X}$-rays often leads to the diagnosis being missed.

\section{Case report}

A 48-year-old woman presented with 4 months of spontaneous progressive pain and limited range of motion of the right shoulder. Her pain was aggravated by movement and impacts, particularly those activities associated with daily living. She was unable to lie on her right side but did not complain of any pain at night. She had no history of trauma although she recently started experiencing episodes of syncope. Further to this, her medical history consisted of hypertension, hypercholesterolaemia, asthma and previous left frozen shoulder.

Upon clinical examination she held the right upper limb in internal rotation with the elbow in flexed position. There was diffuse, mild tenderness over the anterolateral side of the shoulder. Range of motion was limited to 5 degrees of passive and active abduction. External rotation was not possible. Other shoulder tests were impossible to implement. X-rays showed normal proportions of the GH joint with the humeral head articulating in the glenoid. On the anterior side of the humeral head a medium sized dent was visible, suggestive of a reverse Hill-Sachs lesion. The differential diagnosis was an overlooked posterior shoulder dislocation or adhesive capsulitis.

\section{Discussion}

PSD is a rare entity. Incidence is approximately $2-5 \%$ of all shoulder dislocations. ${ }^{[1]}$ Most often PSD is the result of an epileptic seizure. Less frequent causes are seizures due to alcoholic withdrawal or other metabolic disorders, electrocution or during a high-energy trauma. In this particular scenario we suspected that PSD likely occurred as a result of an episode of syncope. According to the literature, PSD is frequently overlooked at the time of injury or may stay clinically undetectable, which makes the real incidence unsure. ${ }^{[2]}$ A missed diagnosis of posterior shoulder dislocation at the time of injury could lead to an incorrect diagnosis of frozen shoulder at a later stage.

Shoulder stability is provided by static and dynamic structures. Posterior static stabilisers of the glenoid are the labrum, the posterior capsule and the posterior band of the inferior GH ligament. Dynamic structures are the shoulder girdle muscles and the rotator cuff. Traumatic PSD occurs when a high axial loading force is applied to the upper extremity in the vulnerable position of internal rotation, adduction and forward elevation. In an involuntary contraction, the strong internal rotators overpower the weak external rotators. The injured extremity is held in internal rotation with the loss of external rotation as the cardinal sign.

Clough and Bale ${ }^{[3]}$ recommend an axillary view of the shoulder if PSD is suspected. An axillary view is taken by gentle abduction of the shoulder to $20-30^{\circ}$ with the X-ray tube at the level of the hip and the cassette at the shoulder. If a true axillary view cannot be taken, usually because it is too painful for the patient, a Velpeau or Wallace view (modified axillary views) is advised.

PSD is regularly accompanied by associated injuries. A fracture of the humeral neck can displace during reduction. The humeral head has a segmental blood supply; if the ascending branch of the arterial humeral circumflex artery is compromised there is a risk for osteonecrosis. ${ }^{[2,4]}$ Other possible fractures involve the greater and lesser tuberosities, posterior rim fracture, or combinations thereof. The most important one is probably the pathognomonic lesion in the humeral head, the reverse Hill-Sachs lesion or encoche fracture. Kowalsky and Levine ${ }^{[1]}$ describe the significance of the humeral head defect as being threefold: it serves as a propagation point which makes the proximal humerus susceptible to fractures of the anatomic neck, it makes the GH joint susceptible to secondary osteoarthritis, and the size of the impression defect determines the stable arc of curvature of the GH articulation. The size of the humeral head impression also has a critical role in the determination of the optimal treatment strategy, as discussed later. Rotator cuff tear injuries are present in $13 \% .{ }^{[5]}$ Less frequent associated injuries are brachial plexopathy and axillary nerve palsy. ${ }^{[6]}$

To date, because of the lack of high-level evidence, there are no 
definitive management guidelines for PSD. An attempt at gentle closed reduction is generally advised if an isolated PSD is less than 6 weeks and the humeral head impression is less than $25 \%$ of the articular surface. In chronic PSD, or if the humeral head impression exceeds $25 \%$ of the articular surface, different operative treatment strategies are described, namely the McLaughlin procedure, modified McLaughlin procedure or a rotation osteotomy of the humerus and reconstruction of the humeral head impression with the use of auto- or allograft. In large defects exceeding more than $50 \%$ of the articular surface of the humeral head or secondary osteoarthritis, an arthroplasty is suggested as being the optimal treatment strategy ${ }^{[7]}$ Owing to the risk of posterior subluxation due to anterior-inferior and subscapularis contractures, an insertion of the prosthesis is made in relatively anteverted orientation, or normal prosthetic retroversion with anterior capsular release. In PSD with the rarer complex fractures of the proximal aspect of the humerus, there have been favourable functional results with the use of open relocation, fracture reduction and stabilisation, and adjuvant stabilisation procedure if necessary in 28 cases. ${ }^{[8]}$ In these complex fracture-dislocations, the majority of the functional loss in the long-term was attributable to loss of internal rotation of the shoulder.

Long-term complications include recurrent instability, osteonecrosis and post-traumatic shoulder stiffness. In a retrospective review of 120 acute, traumatic, isolated posterior GH dislocations Robinson et al. ${ }^{[9]}$ found three factors that were significantly associated with an increased risk of recurrent instability: an age $<40$ years at the time of original dislocation, dislocation during a seizure and the presence of a reverse Hill-Sachs lesion $>1.5 \mathrm{~cm}^{3}$. Therefore, if a redislocation occurs, an additional adjunctive stabilisation procedure is recommended. The same study also underscores the risk for post-traumatic shoulder stiffness after reduction in case of postponed diagnosis, which is probably due to peri-articular fibrosis and capsular contractures.

\section{Conclusion}

This case report demonstrates the importance of taking a proper medical history to rule out syncope as a possible means of trauma giving rise to PSD. Also, while posterior dislocation of the shoulder is generally known among sports medicine practitioners, it is often unrecognised which could lead to the incorrect diagnosis of idiopathic frozen shoulder at a later stage. Diagnosis is primarily based on initial clinical findings and a high index of suspicion after seizure, electrocution or high-energy trauma. One should be aware of associated injuries, especially humeral neck fractures, before attempts for reduction are made. In chronic cases, differential diagnosis includes idiopathic frozen shoulder and a surgical approach is mainly recommended. Post reduction long-term complications involve recurrent instability, osteonecrosis and post-traumatic shoulder stiffness.

\section{References}

1. Kowalsky MS, Levine WN. Traumatic posterior glenohumeral dislocation: Classification, pathoanatomy, diagnosis, and treatment. Orthop Clin North Am 2008;39(4):519-533. [http://dx.doi.org/10.1016/j.ocl.2008.05.008]

2. Robinson CM, Aderinto J. Posterior shoulder dislocations and fracturedislocations. J Bone Joint Surg Am 2005;87(3):639-650. [http://dx.doi. org/10.2106/JBJS.D.02371]

3. Clough TM, Bale RS. Bilateral posterior shoulder dislocation: The importance of the axillary radiographic view. Eur J Emerg Med 2001;8(2):161-163.

4. Hersche $\mathrm{O}$, Gerber $\mathrm{C}$. Iatrogenic displacement of fracture-dislocations of the shoulder: A report of seven cases. J Bone Joint Surg Br 1994;76(1):30-33.

5. Rouleau DM, Hebert-Davies J. Incidence of associated injury in posterior shoulder dislocation: Systematic review of the literature. J Orthop Trauma 2012;26(4):246-251. [http://dx.doi.org/10.1097/BOT.0b013e3182243909]

6. Bhatia DN, de Beer JF, van Rooyen KS, du Toit DF. The reverse terrible triad of the shoulder: Circumferential glenohumeral musculoligamentous disruption and neurologic injury associated with posterior shoulder dislocation. J Shoulder Elbow Surg 2007;16(3):13-17. [http://dx.doi.org/10.1016/j.jse.2006.07.013]

7. Rouleau DM, Hebert-Davies J, Robinson CM. Acute traumatic posterior shoulder dislocation. J Am Acad Orthop Surg 2014;22(3):145-152. [http://dx.doi.org/10.5435/ JAAOS-22-03-145]

8. Robinson CM, Akhtar A, Mitchell M, Beavis C. Complex posterior fracturedislocation of the shoulder: Epidemiology, injury patterns, and results of operative treatment. J Bone Joint Surg Am 2007;89(7):1454-1466. [http://dx.doi.org/10.2106/ JBJS.F.01214]

9. Robinson CM, Seah M, Akhtar MA. The epidemiology, risk of recurrence, and functional outcome after an acute traumatic posterior dislocation of the shoulder. J Bone Joint Surg Am 2011;93(17):1605-1613. [http://dx.doi.org/ 10.2106/ JBJS.J.00973] 